\author{
O. Vasiliev
}

\title{
Thermodynamic Properties of 2H-MoSe 2 From First Principles Quasi-Harmonic Approximation
}

\author{
Frantsevich Institute for Problems of Materials Science NAS of Ukraine, Kyiv, Ukraine, o.vasiliev@outlook.com \\ Igor Sikorsky Kyiv Polytechnic Institute, Kyiv, Ukraine
}

\begin{abstract}
In this paper, we report the results of first-principles calculation of $2 \mathrm{H}-\mathrm{MoSe} 2$ thermodynamic properties within the quasi-harmonic approximation. The focus of the article is on the temperature dependencies of the heat capacity up to $1000{ }^{\circ} \mathrm{C}$ and values of enthalpy of formation, enthalpy, and entropy at $298.15 \mathrm{~K}$, and their comparative analysis with the existing experimental data. The results show good general agreement with the published experimental data sets allowing using them as arbitration of existing discrepancies. Increasing deviations of the heat capacity above the room temperature suggest that factors not included in the quasiharmonic approximation, such as vibrational anharmonicity, may have a significant influence on the thermodynamics of $2 \mathrm{H}-\mathrm{MoSe}_{2}$ in this temperature region. Considering the inconclusive high-temperature data from the experiment, the present results may be recommended as a satisfactory approximation until the appearance of more reliable experimental data or calculation results, taking into account more finite-temperature effects.
\end{abstract}

Keywords: molybdenum diselenide, thermodynamic properties, density-functional theory, quasi-harmonic approximation.

Received March 14, 2020; Accepted September 15, 2020.

\section{Introduction}

The last decade has seen a tremendous increase in scientific interest in layered transition metal dichalcogenides. Initial intensive research into graphene revealed a lot of useful properties that single layer nanoforms may possess, but also showed the material's limitations and instigated the search by the principle of similarity. Group VI transition metal dichalcogenides show the most significant promises among graphene-like materials due to their excellent electronic and optical properties [1]. Hexagonal molybdenum disulfide attracted the most attention so far, probably because of the high natural abundance and existence of a significant volume of research results produced for its bulk form, which had been extensively used as solid lubricant in high-tech applications long before the discovery of graphene. Recently, other dichalcogenides started to get their share of attention and are currently regarded as more promising in some specialized applications. Particularly, 2H-MoSe 2 is expected to have better performance in catalytic, optoelectronic, and energy storage systems due to a larger radius and better metallic properties of selenium atoms than those of sulfur[2].

The indicated application directions for $2 \mathrm{H}-\mathrm{MoSe}_{2}$ require clear understanding and precise data on its thermodynamic properties. Molybdenum diselenide does not occur naturally and must be produced by synthetic routes, most probably using the CVD synthesis (similarly to, for example, [3]). Thermodynamics is among the key factors that determine the ability to control this process to get the product in the desired state. Furthermore, thermodynamic properties of nanoforms of substances may deviate from those of bulk (see, for example, $[4,5]$ ), and the analysis of their energetics and stability demands reliable thermodynamic data.

From the experimental point of view, several groups have investigated the thermodynamic properties of molybdenum disulfide. O'Hare and Tasker in [6] did a fluorine-combustion calorimetric study to determine its standard enthalpy of formation. Their molar $\Delta_{\mathrm{f}} H^{\circ}$ result of $-234.2 \pm 3.3 \mathrm{~kJ} \cdot \mathrm{mol}^{-1}$ at $298.15 \mathrm{~K}$ is in a formal 
agreement with the previous estimate by Mills [7] of $-197 \pm 42 \mathrm{~kJ} \cdot \mathrm{mol}^{-1}$ and the value of $-272.0 \pm 35.0$ obtained by Viksman et al. from the dissociation study[8], but the last two have too wide a range of uncertainty. Kiwia and Westrum in [9] measured the low-temperature heat capacity by adiabatic calorimetry, and Blinder et al. published data in a broad temperature range from adiabatic and drop calorimetry in [10]. The indirect results from the dissociation study on the hightemperature thermodynamic properties are also given in [8].

The results of a theoretical study using current capabilities of the Density Functional Theory would undoubtedly increase the reliability of the existing dataset, as well as improve the understanding of specific influences on the thermodynamic properties of $2 \mathrm{H}$ $\mathrm{MoSe}_{2}$ on an atomic scale. As per our knowledge, very little has been done to address this issue. Sevik in [11] used first-principle calculations and quasi-harmonic approximation to calculate phonon spectra and thermal expansion coefficients of several layered structures, including $\mathrm{MoSe}_{2}$, but the article does not discuss other thermodynamic properties or provide any data on them. Ding and Xiao in [12] similarly focus on the phonon spectra, Grüneisen parameters, and thermal expansion. Several works are addressing thermal transport in bulk and single to few-layer $\mathrm{MoSe}_{2}$ using similar computational techniques, the references for which we do not provide here considering their distant relevance. An interested reader can find them using any of the conventional reference search engines.

The objective of this work is to calculate the heat capacity and standard values of enthalpy of formation, enthalpy, and entropy of $2 \mathrm{H}-\mathrm{MoSe}_{2}$ from first principles using the quasi-harmonic approximation to lattice vibrations and analyze the input of results into the reliability of the set of data available from experimental research.

\section{Details of calculation}

Temperature-dependent constant-volume thermodynamic properties in this work were calculated using the finite displacement technique and supercells, as realized in the phonopy software[13]. The method uses predetermined small displacements of atoms from their equilibrium position to calculate the second-order force constants from the first principles, build the dynamical matrix, determine phonon dispersion relations and density of states, and calculate thermodynamic properties using statistical thermodynamics. The supercell size of $2 \times 2 \times 1$ unit cells was chosen for the calculation, so that its shape was as close to cubic as possible, considering the unit cell geometry. Atomic displacements in the unit cell were 0.02 au (Bohr).

Gibb's free energy, thermal expansion, and the heat capacity at constant pressure of $2 \mathrm{H}-\mathrm{MoSe}_{2}$ were calculated using the Quasi-Harmonic Approximation (QHA) to thermal vibrations in solids. Using this approximation allows calculating a part of the finitetemperature effect caused by the change of phonon frequencies with the increase of equilibrium unit cell volume. As this effect is the dominant factor in anharmonicity of most solids at low and moderate temperatures, the QHA may suffice for the calculation of thermodynamic properties at constant pressure with accuracy within the margins of experimental studies.

For the QHA calculations, total electronic energies $E_{\text {tot }}$ at zero temperature and pressure and vibrational free energies $F_{\text {vib }}$ were calculated at 11 unit cell volumes, ranging from 0.95 to 1.05 of the relaxed at zero temperature and pressure unit cell, and fitted to the Vinet equation of state (EOS)[14]. Using the EOS, Gibbs free energies at specific finite temperature $T$ and pressure $p$ were calculated as minimums with respect to volume

$G(T, p)=\min f(V ; T, p)=\min \left[E_{\mathrm{tot}}(V)+F_{\mathrm{vib}}(V ; T)+p V\right]$,

and the minimizing volume was considered to be the equilibrium volume at this temperature and pressure. The constant pressure heat capacity was then calculated by numerical differentiation of the Gibbs free energy with respect to temperature and thermal expansion as an equilibrium volume of the unit cell increase. The fitting and calculation of isobaric thermodynamic functions were done using the phonopy-qha script from the phonopy software package.

$A b$-initio calculations of total electronic energies and interatomic forces in displaced supercells were done using the Density Functional Theory (DFT)[15] as implemented in the PWscf code of the Quantum Espresso ( $Q E)$ package[16,17]. For the exchangecorrelation functional the Local Density Approximation (LDA) [18] was used, as it showed better results than Generalized Gradient Approximation (both PBE [19] and PBEsol [20]) in testing and was reported to be suitable for layered structures with van der Waals bonding[21,22]. Specifically, the calculation was done with ultra-soft pseudopotentials from the $Q E$ library. Kinetic energy cut-off for the basis plane waves was set to $816 \mathrm{eV}(60 \mathrm{Ry})$ in all calculations, as it was sufficient for the total energy convergence within $1 \mathrm{meV} / \mathrm{atom}$. The Brillouin zone integrations were done using the grids of special points set according to the Monkhorst-Pack (MP) scheme [23]. Settings for the grids for each structure, which are given below with the structure descriptions, provided total energy convergence below $1 \mathrm{meV} /$ atom. All the initial structures were relaxed until forces in the unit cells were less than $10^{-4} \mathrm{eV} / \mathrm{a} . u$.

Standard enthalpy of formation of the hexagonal molybdenum diselenide $\left(\Delta_{\mathrm{f}} H^{\circ}\left(2 \mathrm{H}-\mathrm{MoSe}_{2}\right)\right)$ was calculated as the energy of reaction of its formation from elements in their reference states:

$$
\mathrm{Mo}(\mathrm{s})+2 \mathrm{Se}(\mathrm{s}) \rightarrow \mathrm{MoSe}_{2}(\mathrm{~s}, 2 \mathrm{H})
$$

which is:

$\Delta_{\mathrm{f}} H^{\circ}\left(2 \mathrm{H}-\mathrm{MoSe}_{2}, 0 \mathrm{~K}\right)=E_{\mathrm{tot}}\left(2 \mathrm{H}-\mathrm{MoSe}_{2}\right)-\left(E_{\mathrm{tot}}(\mathrm{Mo})+2 E_{\mathrm{tot}}(\mathrm{Se})\right)$, 
where $E_{\mathrm{tot}}(\mathrm{X})$ is the DFT total energy per mol of formula units of species $X$.

Total energy calculations in DFT are done at zero temperature and pressure; therefore, the energy of reaction (1) technically is the change of internal energy. Because of small molar volumes of solids, the difference between internal energy and enthalpy for them is several orders of magnitude lower than typical experimental errors in calorimetry. Thus, for most practical purposes, eq. (2) is a reasonable approximation. Additionally, standard enthalpy of formation is typically reported at $T=289.15 \mathrm{~K}$. To get such a value from the formation enthalpy at $0 \mathrm{~K}$, vibrational corrections to the enthalpy (enthalpy increase due to thermal excitations of the vibrational spectrum) of all species in the reaction (1) must be accounted. As all the participants of reaction (1) are solids, temperature effect on their total energy up to room temperatures is close and practically cancels out, which was shown in [22].

The $2 \mathrm{H}$ modification of molybdenum diselenide has a layered structure with Se-Mo-Se "sandwiches" separated by van der Waals gaps (Figure 1). Its hexagonal unit cell belongs to the $P 6_{3} / m m c$ (194) space group. It contains two formula units, one per each layer, and has lattice parameters $a=b=0.3288 \mathrm{~nm}$, $c=1.2931 \mathrm{~nm}, \quad \alpha=\beta=90^{\circ}, \quad \gamma=120^{\circ}$. Molybdenum atoms reside at $\pm(1 / 3,2 / 3,1 / 4)$ and $\mathrm{Se}$ at $\pm(1 / 3,2 / 3, \mu)$ and $\pm(2 / 3,1 / 3, \mu+1 / 2)$ with $\mu \approx 5 / 8$, as refined from the $\mathrm{X}$-ray diffraction in [24]. The MP grid for Brillouin zone integration was $8 \times 8 \times 2$ points displaced by a half-step in $a$ and $b$ directions.

Molybdenum crystalizes into body-centered cubic structure, space group Im-3m (229), with two atoms per unit cell and experimental values of lattice parameters $a=b=c=0.31473 \mathrm{~nm}$ and $\alpha=\beta=\gamma=90^{\circ}$. The Brillouin zone integrations were done on a grid of $8 \times 8 \times 8$.

Trigonal selenium was used as the reference state of Se in this work, as accepted by the scientific community [25]. Its hexagonal conventional unit cell has lattice parameters $a=0.43662$ and $c=0.49536 \mathrm{~nm}$, contains three atoms lying on twofold axes, and belongs to the $P 3_{1} 21$ (152) space group. The MP grid for the Se total energy calculation was $8 \times 8 \times 8$ displaced by half-step in $a$ and $b$ directions.

\section{Results and discussion}

\section{Structure relaxation}

All the structures considered in this work were relaxed until the total force acting on atoms of the unit cell was smaller than $1 \cdot 10^{-4} \mathrm{eV} / \mathrm{a} . \mathrm{u}$. While this is a standard procedure in DFT calculations, we paid additional attention to get a good force convergence, as even small artificial (for a given exchange-correlation approximation) deviations from equilibrium geometry can lead to significant errors in the force constants and, thus, in thermodynamic properties. It is worth noting that sometimes experimental geometry may give a better agreement of results with an experiment, as was shown in [22]. It may be especially true in the case of enthalpy of formation values. So, iIn this work, this quantity was
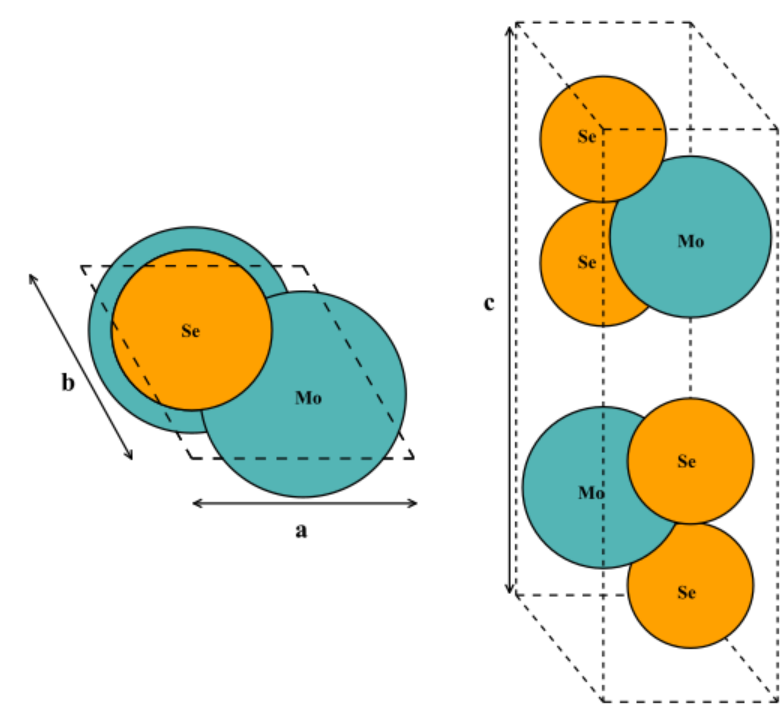

Fig. 1. Unit cell of $2 \mathrm{H}-\mathrm{MoSe}_{2}$. Lattice parameters for the experimental and relaxed structure are in the text.

calculated from total energies of both experimental and relaxed structures.

Table 1 shows the lattice parameters for all the involved structures before and after the relaxation. There are considerable deviations from the experimental structure for each compound, as well as in different crystallographic directions. The apparent general reason for this is how LDA treats different types of chemical bonding, though this explanation does not work for each case.

Negative deviations for the relaxed structures which demonstrate bond "shrinkage" (or rather an underestimation) are the result of the expected behavior of LDA. It tends to overestimate the strength of the bonds, making them shorter, and it seems the weaker is the bond, the more it contracts. It is the primary reason why deviations for the a direction in Se and c in $\mathrm{MoSe}_{2}$, both including weak van der Waals bonding, are large, while for strong metallic bonds in Mo and covalent in a direction in $\mathrm{MoSe}_{2}$ the agreement is better. Positive deviation for the covalently bonded direction $\mathrm{c}$ in elemental Se is, however, an unexpected result. This anomalous expansion was checked with various flavors of LDA pseudopotentials, and it is present in every case and thus requires attention in further works. For the current work, all the indicated deviations may significantly influence the results for the enthalpy of formation.

Table 1

Relaxed lattice parameters of Mo, Se, and 2H-MoSe ${ }_{2}$

\begin{tabular}{|c|c|c|}
\hline Structure & $\begin{array}{c}\text { Relaxed lattice } \\
\text { parameters, } \mathrm{nm}\end{array}$ & $\begin{array}{c}\text { Deviation from } \\
\text { experiment, \% }\end{array}$ \\
\hline Mo (bcc) & $a=0.31198$ & -0.87 \\
\hline \multirow{2}{*}{$\mathrm{Se}$ (trigonal) } & $a=0.39404$ & -9.75 \\
& $c=0.51322$ & 3.61 \\
\hline \multirow{2}{*}{$\mathrm{MoSe}_{2}(2 \mathrm{H})$} & $\mathrm{a}=0.32607$ & -0.83 \\
& $\mathrm{c}=1.27429$ & -1.45 \\
\hline
\end{tabular}

It is worth noting, that deviations from the experimental geometry in LDA serve, to some extent, as 
an "empirical device" for taking into account the weak interlayer bonding in $2 \mathrm{H}-\mathrm{MoSe}_{2}$ in the force-constants calculation. Most approximations for the exchangecorrelation functional in DFT do not take into account intermolecular interactions by any particular means. Underestimation of a bond length and, consequently, overestimation of its energy by the LDA accidentally accounts for the van der Waals bonding. The effect is not physical and is not theoretically reflected in the approximation itself, and there is no explicit correlation between the real energy of interaction and what is taken into account. On the other hand, when it leads to better agreement between the experiment and calculation, this way is, probably, the most useful when using purely firstprinciple well-tested approaches.

Despite the difference in lattice parameters for the relaxed structure, our tests with several pseudopotential types showed that LDA is the most suitable for $2 \mathrm{H}$ $\mathrm{MoSe}_{2}$ vibrational properties calculations (criteria: the quality of agreement between calculated and experimental low-temperature heat capacity). Generalized gradient approximation adapted for solids showed the most reliable results for the total energy and was also used for enthalpy of formation calculation.

\section{Phonon spectrum}

Calculated phonon dispersions and density of states are given in Figure 2, which demonstrates good agreement of the phonon frequencies in the Brillouin zone center with experimental data from Raman spectroscopy [26]. While the agreement suggests that the calculated phonon spectrum is correct near the zone center, we could not find any neutron diffraction data to check against for the directions between high symmetry points. Given the situation, we considered these dispersions and phonon density of states based on them as appropriate for the calculation of thermodynamic properties.

Phonon spectrum (dispersions) of $2 \mathrm{H}-\mathrm{MoSe}_{2}$ have a rather peculiar structure with distinct splitting between the high-energy optical modes. As it has limited relevance in the context of integral thermodynamic quantities, only to the extent it influences the phonon DOS, its discussion is left out to keep the present text succinct. An interested reader might find some details on it in, for example [12]. From the general view of the phonon DOS, we can expect a steady climb of the heat capacity values at low and moderate temperatures with the majority of vibrations, including a part of the optical spectrum, already excited at just above the room temperature. However, an asymptotic approach of the limiting value can be expected to extend into a broad region at high temperatures due to the excitation of the rest of optical vibrations.

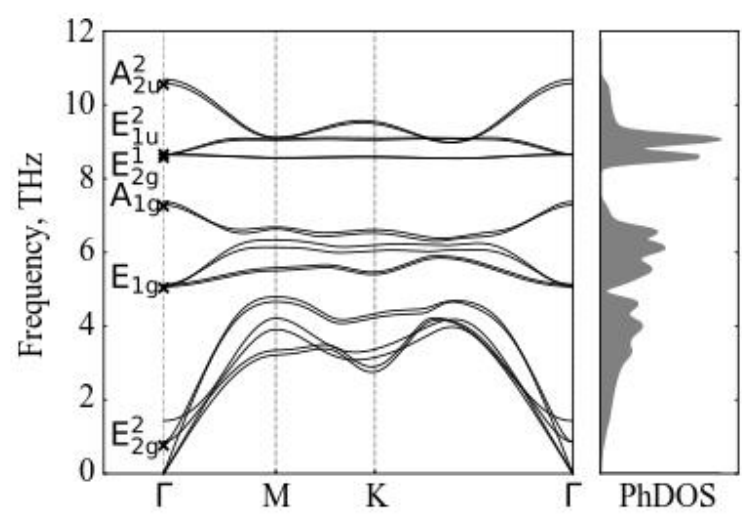

Fig. 2. Phonon dispersions and density of states of $2 \mathrm{H}-$ $\mathrm{MoSe}_{2}$ : solid line - this work (calculated), $\times$ - experimental Raman-active data [26].

Quasi-harmonic approximation in solids allows obtaining of several important properties associated with vibrational energy. Among them is the information on the equilibrium unit cell volume at specific finite temperatures, volumetric thermal expansion coefficient, Gibb's free energy, and constant pressure heat capacity. For some of these properties, there are experimental results in published sources, which allow the assessment of the validity of approximation at several stages of calculation.

Figure 3 shows the results of free energy minimization to find the equilibrium unit cell volume at several temperatures (a), the temperature dependence of the molar (unit cell) volume (b), and the volumetric thermal expansion coefficient (c). While section (a) of
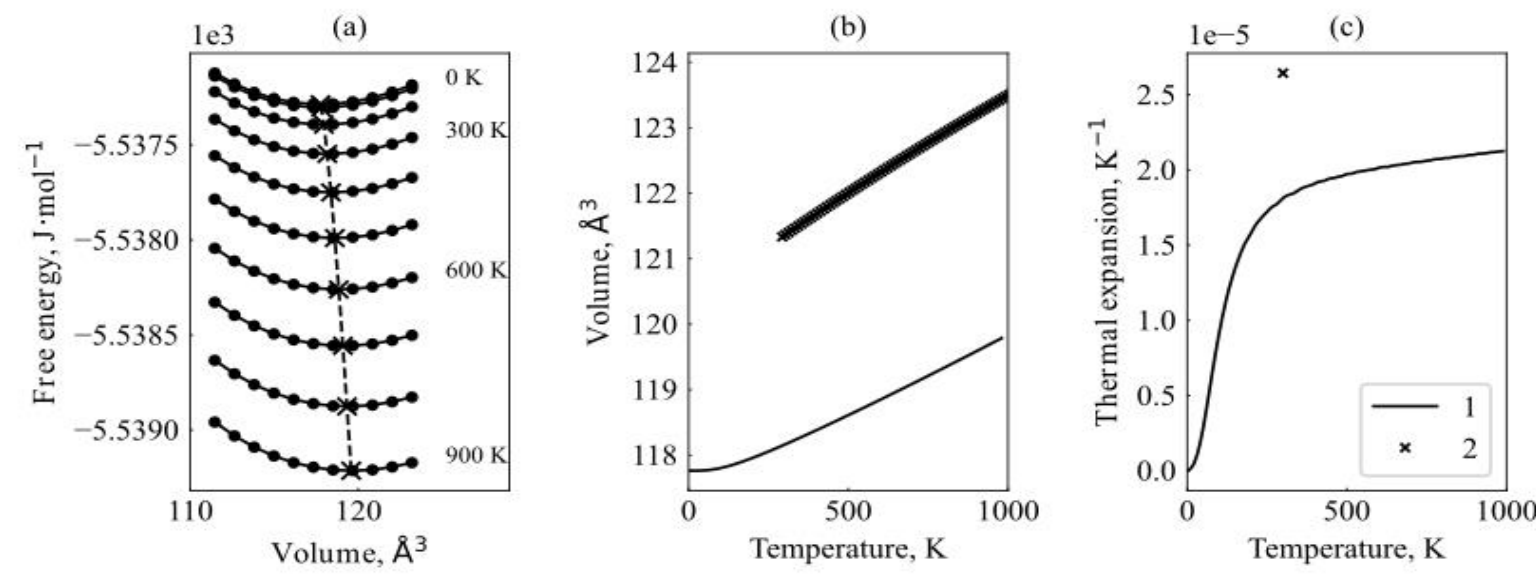

Fig. 3. Equilibrium volume and thermal expansion of $2 \mathrm{H}-\mathrm{MoSe}_{2}$ : $\mathrm{a}$ - free energy minimization; $\mathrm{b}$ - temperature dependence of equilibrium volume; $\mathrm{c}$ - temperature dependence of the thermal expansion coefficient. In sections b and c: 1 - present work, $2-$ [27]. 
the figure serves purely demonstrative purposes showing the free energy minimization results, sections (b) and (c) show the results of equilibrium volume and thermal expansion calculation respectively, together with some available experimental data [27]. As can be seen from Figure 3(b), equilibrium volume stays practically constant up to around $100 \mathrm{~K}$. Such behavior may be explained by the primary excitation of interplanar lowenergy vibrations up to these temperatures. Because of the significant distance between the planes, the atomic mean-square displacements from equilibrium positions in $2 \mathrm{H}-\mathrm{MoSe}_{2}$ may be significant without much mutual influence of the atoms in the adjacent layers. Therefore, the increase in equilibrium volume is expected to happen when intralayer vibrations start to be excited to a considerable extent. The volumetric thermal expansion coefficient (Figure 3(c)), as a measure of the lattice volume increase, rises steeply up to around room temperature and asymptotically approaches its limiting value above it, seemingly contradicting the equilibrium volume pattern. Its low absolute values lead to a considerable increase in volume only after the limiting value is already almost reached, at around $300 \mathrm{~K}$, which reconciles the two datasets.

An apparent significant disagreement between the experimental data and calculated values of equilibrium volume and coefficient of thermal expansion requires some additional attention from the standpoint of absolute values analysis and justification of experimental and computational procedures. Unfortunately, the use of local density approximation in this work does not allow obtaining of reliable absolute values for equilibrium volume but the differences here reflect the "shrinkage" of the unit cell in relaxation. The rate of volume increase represented by the thermal expansion coefficient, on the other hand, should be representative of the material's behavior irrespectively of absolute values of volume. Figure 2(c) demonstrates that this is true to a large extent - the deviation between the experimental and calculated thermal expansion coefficients is within one order of magnitude, which can be considered a good agreement, taking into account the complexity of the determination of this quantity using X-ray diffraction and small absolute values. As can be seen from the overview of different experimental results in [27], the deviations between different experimental sets may even be more significant than the difference between experimental and present calculated results.

On the other hand, the quasi-harmonic approximation does not consider any real anharmonicity of vibrations in a crystal lattice. It may be a significant source of discrepancy between the measurements on real materials and calculations on ideal structure, especially above the room temperature, where anharmonicity may be substantial. Thus, the deviation of thermal expansion coefficients is the first indication that the hightemperature thermodynamic properties should be studied using approaches taking into account finite temperature effects more physically.

\section{Thermodynamic properties}

In this work, we take a typical experimental approach to the results, where the heat capacity at constant pressure plays a central role, despite it being only a derivative of the Gibbs free energy in the calculations. Figure 4 shows the results of the calculation of heat capacity at constant volume and pressure together with available experimental data. Up to around $180 \mathrm{~K}$, the results of calculation and experiment are in good agreement, especially with experimental data [9]. The irregular shape of the temperature dependence of the heat capacity in the region $180-200 \mathrm{~K}$ in [10] is most

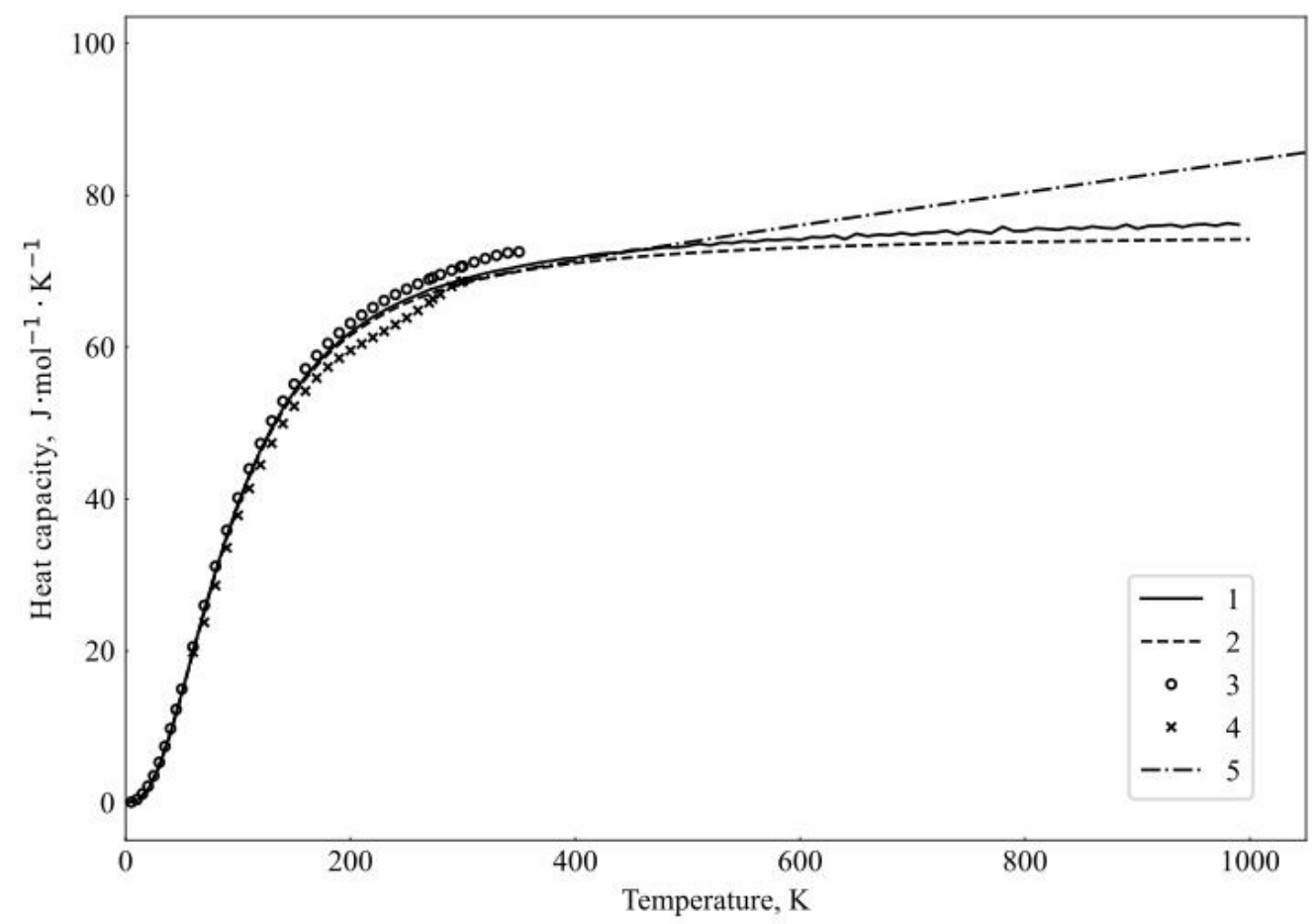

Fig. 4. Molar heat capacity of $2 \mathrm{H}-\mathrm{MoSe}_{2}$ : 1 - constant pressure (present work); 2 - constant volume (present work); 3 - [9]; 4 - adiabatic calorimetry from [10]; 5 - drop-calorimetry from [10]. 
probably the result of unsatisfactory experimental procedure execution, but the room temperature value also agrees well with the calculation results. It is important to note that the values obtained from calculation lay inbetween the experiments, which allows using them as a kind of arbitration for the two sets.

In contrast, the high-temperature data derived from the polynomial expressions in [10] is not by any means conclusive. The polynomial is tied to the experimental data at low temperatures, and the heat capacity values it describes acceptably agree with the calculations at around room temperature, but their further increase is too steep. As these data are derived from the results of the experimental determination of enthalpy, some significant deviation is expected, it may reach up to $20 \%$ or more. However, the character of temperature dependence at very high temperatures is incorrect. Low-temperature data suggest that it should plateau instead of increasing linearly. On the other hand, the results at room temperature in [9] indicate that at high temperatures, the agreement of calculations and experiment would not be as good as it is at low temperatures. It is a second sign, after the thermal expansion coefficient, indicating that the quasi-harmonic approximation may be insufficient to describe high-temperature thermodynamic properties reliably.

Calculated temperature dependencies of the heat capacity at constant volume and constant pressure are mostly determined by the specifics of the phonon spectrum and thermal expansion in quasi-harmonic approximation. Values of isobaric and isochoric heat capacities at temperatures up to $200 \mathrm{~K}$ practically coincide, and some visible deviations due to thermal expansion can be seen only at higher temperatures. The differences between the two sets of data do not exceed those between experimental values in different studies. On the other hand, considering the specifics of taking into account the anharmonicity of vibrations in quasiharmonic approximation and the agreement with experimental data [9] at room temperatures, the input into heat capacity due to thermal expansion is probably higher already at room temperatures and increases with temperature. Thus, establishing the high-temperature heat capacity data that is closer to its real values requires additional studies. Computational methods are indispensable in this case considering the significant errors of experimental measurements at elevated temperatures,

Acceptable agreement of the calculation results with experimental data in the $0-300 \mathrm{~K}$ region allows using them and the calculated Gibbs free energy to obtain standard values of thermodynamic properties of $2 \mathrm{H}-\mathrm{MoSe}_{2}$, which are given in Table 2 together with literature data for comparison.

Calculation of the enthalpy of formation $\Delta_{\mathrm{f}} \mathrm{H}^{\circ}(\mathrm{T})$ is a part of work not connected with the vibrational properties, as it is done using only the total energies of the compound and elements forming it in their ground states $(\mathrm{p}=0, \mathrm{~T}=0)$. We attempted to calculate it using the experimental crystal lattice, the structure after the relaxation, but also using alternative approximations to the exchange-correlation functional. As can be seen from Table 2, all the results exceed experimental by more than $10 \%$. When using local density approximation as the exchange-correlation functional, the case of unrelaxed structure comes closest to the experimental data, similar to [22]. Using generalized gradient approximation adapted for solids (PBEsol) for the exchange-correlation functional gives the best results. Typical deviations between the results of experimental determination of the enthalpy of formation in different works often reach more than $25 \%$, and data within less than $15-20 \%$ are considered to be in good agreement. Thus, the results of the present work are suitable for further use.

Other thermodynamic properties in Table 2 demonstrate a similar pattern - the results of calculation lay between the experimental values by different authors. In statistical assessment, they are the closest to the mean and thus have high reliability. It allows recommending them for the use in thermodynamic databases, thermodynamic calculations, and analysis of real processes.

In conclusion, the calculation of the thermodynamic properties of $2 \mathrm{H}-\mathrm{MoSe}_{2}$ from the first principles in quasiharmonic approximation provides a valuable addition to the statistics of their standard values at $298.15 \mathrm{~K}$, which is essential for further thermodynamic analysis of the synthesis of layered molybdenum disulfide and materials based on it. An increase in their reliability resulting from this work leads to more precise analysis, which is needed when working with nanoforms of these materials. Despite this, the quasi-harmonic approximation seems to be incapable of taking into account all the temperature effects on the thermodynamics. In particular, the input

Table 2

Standard values of the thermodynamic function of $2 \mathrm{H}-\mathrm{MoS}_{2}(T=298,15 \mathrm{~K})$

\begin{tabular}{|c|c|c|c|}
\hline Function & Calculation & Experiment & Deviation, $\%$ \\
\hline$\Delta_{\mathrm{f}} H^{\circ}(T), \mathrm{kJ} \cdot \mathrm{mol}^{-1}$ & $\begin{array}{c}-201 \text { (expt. lattice) } \\
\text {-198 (relaxed lattice) } \\
\text {-207 (PBEsol) }\end{array}$ & $-234.2 \pm 3.3[6]$ & $\begin{array}{c}14 \\
15 \\
11.6\end{array}$ \\
\hline$C_{\mathrm{p}}{ }^{\circ}(T), \mathrm{J} \cdot \mathrm{mol}^{-1} \cdot \mathrm{K}^{-1}$ & 69.25 & $\begin{array}{l}68.6[10] \\
70.58[9]\end{array}$ & $\begin{array}{c}0.94 \\
-1.92\end{array}$ \\
\hline$S^{\circ}(T), \mathrm{J} \cdot \mathrm{mol}^{-1} \cdot \mathrm{K}^{-1}$ & 86.05 & $\begin{array}{l}83.3[10] \\
89.08 \text { [9] }\end{array}$ & $\begin{array}{c}3.19 \\
-3.52\end{array}$ \\
\hline$H^{\circ}(T)-H^{\circ}(0), \mathrm{J} \cdot \mathrm{mol}^{-1}$ & 13516 & $\begin{array}{c}12930[10] \\
13670[9]\end{array}$ & $\begin{array}{c}4.3 \\
-1.1\end{array}$ \\
\hline
\end{tabular}


from anharmonic vibrations comes out underestimated. Obtaining reliable values of thermodynamic properties above the room temperature requires alternative approaches to calculations, such as temperaturedependent effective potential or self-consistent phonons methods.
This work was done with financial support from the NAS of Ukraine under Grant no. III-7-17.

Vasiliev O. - Associate Professor, Ph.D. (Candidate of Chemical Sciences), Senior Researcher.

[1] M. Samadi, N. Sarikhani, M. Zirak, H. Zhang, H.-L. Zhang, A.Z. Moshfegh, Nanoscale Horiz., 3, 90 (2018) (https://doi.org/10.1039/C7NH00137A).

[2] A. Eftekhari, Applied Materials Today. 8, 1 (2017) (https://doi.org/10.1016/j.apmt.2017.01.006).

[3] I.G. Vasilyeva, I.P. Asanov, L.M. Kulikov, J. Phys. Chem. C. 119, 23259 (2015) (https://doi.org/10.1021/acs.jpcc.5b07485).

[4] V.B. Muratov, O.O. Vasil'ev, L.M. Kulikov, V.V. Garbuz, Yu.V. Nesterenko, T.I. Duda, J. Superhard Mater., 34, 173 (2012) (https://doi.org/10.3103/S1063457612030045).

[5] O.O. Vasiliev, V.B. Muratov, L.M. Kulikov, V.V. Garbuz, T.I. Duda, J. Superhard Mater., 37, 388 (2015) (https://doi.org/10.3103/S1063457615060039).

[6] P.A.G. O’Hare, I.R. Tasker, J.M. Tarascon, The Journal of Chemical Thermodynamics. 19, 61 (1987) (https://doi.org/10.1016/0021-9614(87)90163-7).

[7] K.C. Mills, Thermodynamic data for inorganic sulphides, selenides and tellurides (Butterworths, London, 1974).

[8] G.Sh. Viksman, S.P. Gordienko, A.A. Yanaki, L.A. Klochkov, Soviet Powder Metallurgy and Metal Ceramics 25, 64 (1986) (https://doi.org/10.1007/BF00843024).

[9] H.L. Kiwia, E.F. Westrum, The Journal of Chemical Thermodynamics 7, 683 (1975) (https://doi.org/10.1016/0021-9614(75)90009-9).

[10] A.V. Blinder, A.S. Bolgar, Zh.A. Trofimova, Powder Metall. Met. Ceram. 32, 234 (1993) (https://doi.org/10.1007/BF00559756).

[11] C. Sevik, Phys. Rev. B., 89, 035422 (2014) (https://doi.org/10.1103/PhysRevB.89.035422).

[12] Y. Ding, B. Xiao, RSC Adv. 5, 18391 (2015) (https://doi.org/10.1039/C4RA16966B).

[13] A. Togo, I. Tanaka, Scripta Materialia 108, 1 (2015) (https://doi.org/10.1016/j.scriptamat.2015.07.021).

[14] P. Vinet, J.H. Rose, J. Ferrante, J.R. Smith, J. Phys.: Condens. Matter. 1, 1941 (1989) (https://doi.org/10.1088/0953-8984/1/11/002).

[15] P. Hohenberg, W. Kohn, Phys. Rev., 136, B864 (1964) (https://doi.org/10.1103/PhysRev.136.B864).

[16] S. Baroni, P. Giannozzi, A. Testa, Phys. Rev. Lett. 58, 1861 (https://doi.org/10.1103/PhysRevLett.58.1861).

[17] P. Giannozzi, O. Andreussi, T. Brumme, O. Bunau, M. Buongiorno Nardelli, M. Calandra, R. Car, C. Cavazzoni, D. Ceresoli, M. Cococcioni, N. Colonna, I. Carnimeo, A. Dal Corso, S. de Gironcoli, P. Delugas, R.A. DiStasio, A. Ferretti, A. Floris, G. Fratesi, G. Fugallo, R. Gebauer, U. Gerstmann, F. Giustino, T. Gorni, J. Jia, M. Kawamura, H.-Y. Ko, A. Kokalj, E. Küçükbenli, M. Lazzeri, M. Marsili, N. Marzari, F. Mauri, N.L. Nguyen, H.-V. Nguyen, A. Otero-de-la-Roza, L. Paulatto, S. Poncé, D. Rocca, R. Sabatini, B. Santra, M. Schlipf, A.P. Seitsonen, A. Smogunov, I. Timrov, T. Thonhauser, P. Umari, N. Vast, X. Wu, S. Baroni, J. Phys.: Condens. Matter. 29, 465901 (2017) (https://doi.org/10.1088/1361-648X/aa8f79).

[18] J.P. Perdew, A. Zunger, Phys. Rev. B., 23, 5048 (1981) (https://doi.org/10.1103/PhysRevB.23.5048).

[19] J.P. Perdew, K. Burke, M. Ernzerhof, Phys. Rev. Lett. 77, 3865 (1996) (https://doi.org/10.1103/PhysRevLett.77.3865).

[20] J.P. Perdew, A. Ruzsinszky, G.I. Csonka, O.A. Vydrov, G.E. Scuseria, L.A. Constantin, X. Zhou, K. Burke, Phys. Rev. Lett. 100, 136406 (2008) (https://doi.org/10.1103/PhysRevLett.100.136406).

[21] A. Molina-Sánchez, L. Wirtz, Phys. Rev. $\quad$ B, $84, \quad 155413 \quad$ (2011) (https://doi.org/10.1103/PhysRevB.84.155413).

[22] O.O. Vasiliev, Powder Metall. Met. Ceram. 58, 230 (2019) (https://doi.org/10.1007/s11106-019-00068-x).

[23] H.J. Monkhorst, J.D. Pack, Phys. Rev. B., 13, 5188 (1976) (https://doi.org/10.1103/PhysRevB.13.5188).

[24] P.B. James, M.T. Lavik, Acta Cryst. 16, 1183 (1963) (https://doi.org/10.1107/S0365110X6300311X).

[25] A. Olin, F.J. Mompean, Chemical thermodynamics of selenium (Elsevier, Amsterdam, 2005).

[26] T. Sekine, M. Izumi, T. Nakashizu, K. Uchinokura, E. Matsuura, J. Phys. Soc. Jpn. 49, 1069 (1980) (https://doi.org/10.1143/JPSJ.49.1069).

[27] S.H. El-Mahalawy, B.L. Evans, J Appl Crystallogr. 9, 403 (https://doi.org/10.1107/S0021889876011709). 


\title{
О. Васільєв
}

\section{Термодинамічні властивості 2H-MoSe 3 перших принципів та квазігармонійного наближення}

\author{
Інститут проблем матеріалознавства ім. I. М. Францевича НАН Украйни, \\ Київ, Україна, o.vasiliev@outlook.com \\ Національний технічний університет Украӥни «Київський політехнічний інститут імені Ігоря Сікорського», \\ Київ, Україна
}

\begin{abstract}
У статті подані результати розрахунку термодинамічних властивостей $2 \mathrm{H}-\mathrm{MoSe}_{2} 3$ перших принципів у квазі-гармонійному наближенні. Увага у роботі зосереджена на температурних залежностях теплоємності до $1000{ }^{\circ} \mathrm{C}$, значеннях ентальпії утворення, ентальпії та ентропії речовини за 298,15 K, а також їх порівнянні з існуючими експериментальними даними. Результати, в цілому, демонструють добре узгодження з опублікованими даним з експерименту і можуть бути використані в якості арбітражних для оцінки відмінностей, що існують. Видиме зростання відмінностей розрахованої та експериментальної теплоємності за температур понад кімнатною дозволяє припустити, що фактори не враховані у квазігармонійному наближенні, наприклад, вібраційний ангармонізм, можуть суттєво впливати на термодинамічні властивості $2 \mathrm{H}-\mathrm{MoSe}_{2}$ у цьому температурному інтервалі. Зважаючи на невисоку достовірність високотемпературних експериментальних даних, результати даного розрахунку можуть бути рекомендовані в якості задовільного наближення до появи надійних даних з експерименту або розрахунку, який приймає до уваги ефекти скінченних температур в більш повній мірі.

Ключові слова: диселенід молібдену, термодинамічні властивості, теорія функціоналу електронної густини, квазігармонійне наближення.
\end{abstract}

